\title{
Interleukin-6: A Potential Salivary Biomarker for Dental Caries Progression-A Cross-sectional Study
}

\author{
Kiranmayi Govula ${ }^{1}$, Lavanya Anumula ${ }^{2}$, Sannapureddy Swapna ${ }^{3}$, Richard Kirubakaran ${ }^{4}$
}

\begin{abstract}
Background: Resistance or susceptibility to caries is significantly correlated with the alterations in salivary proteins and cytokines, a useful biomarker in predicting caries' risk and prognosis. Bacteria colonize the oral cavity and lead to inflammation, which induces both innate and adaptive immune responses by the host. A predominant cell of inflammation like T lymphocytes, monocytes, macrophages, and endothelial cells expresses many pro-inflammatory cytokines. Interleukin-6 (IL-6) is a multifunctional cytokine transiently produced in response to tissue injury and infections, thus correlating with the extent of inflammation and edema in the pulp. Studies have revealed a correlation between IL- 6 levels and different pulpal conditions, which can contribute to the early diagnosis of pulpal inflammation in caries exposures.

Aim and objective: To assess and compare the salivary IL-6 levels in patients before and after caries removal.

Materials and methods: A total of 20 patients have participated in this study based on inclusion criteria. A pretreatment saliva (first) sample was collected, centrifuged, and stored at $-80^{\circ} \mathrm{C}$. Based on the clinical examination and investigations, the treatment plan was formulated for each patient. After oral prophylaxis and restorative treatment, the patients were followed up after 45 days. The posttreatment (second) saliva samples were collected, and both the samples (first and second) were subjected to the ELISA test to measure the IL-6 levels.

Results: The obtained optical density values were statistically analyzed using the Wilcoxon signed-rank test. As estimated in this study, the mean salivary IL-6 level was $15.32 \pm 17.54$, which reduced to $4.19 \pm 1.45$ after 45 days or one and half months of treatment.

Conclusion: The levels of IL-6 reduced significantly after the complete removal of caries and restoring those teeth with restorative materials showing that there exists a strong correlation between the two.

Clinical significance: A strong correlation is present between the IL- 6 level and the extent and severity of the carious lesions. Posttreatment, there is a significant reduction of the IL- 6 values, which can be attributed to the complete removal of dental caries.

Keywords: Cytokines, Dental caries, ELISA, Interleukin 6, Saliva.

International Journal of Experimental Dental Science (2021): 10.5005/jp-journals-10029-1220
\end{abstract}

\section{INTRODUCTION}

Dental caries from decades is an existing disease in the community. It is known as a multifactorial infectious disease that is mainly caused by complex interactions. The primary mechanism of dental caries can be understood by analyzing the dynamic balance between pathological factors such as acid-producing bacteria, fermentable carbohydrates, host factors, and protective factors such as fluoride, calcium, and phosphates. Among the pathological factors, the organic and inorganic components of saliva play an essential role in bacterial colonization and elimination from the oral cavity. ${ }^{1}$ It is a well-known fact that bacterial colonization in the oral cavity leads to inflammation, which induces innate and adaptive host immune responses. Many interactions occur between various cytokines at the inflammatory sites, responsible for the host immune response shift. Hence, cytokines act as an excellent inflammatory biomarker in the saliva. ${ }^{2}$

Cytokines are the modulators of the immune and inflammatory response and other factors. They will help to diagnose and monitor various oral cavity diseases. Among these cytokines, interleukin-6 (IL-6) has numerous biological effects and acts as a significant mediator of the host response during tissue injury, infection, and inflammation. Interleukin 6 accelerates pulpal inflammation and edema by an increase in vascular permeability. Also, it induces osteoclasts differentiation and bone resorption. ${ }^{3}$

Interleukin 6 has emerged as one of the network mediators that directs the shift from innate to specific immunity through differential control of leukocyte recruitment, activation, and apoptosis. The \begin{tabular}{l}
\hline${ }^{1-3}$ Department of Conservative Dentistry and Endodontics, Narayana \\
Dental College and Hospital, Dr NTR University of Health Sciences,
\end{tabular} Nellore, Andhra Pradesh, India

${ }^{4}$ Department of Biostatistics, BVMC, Christian Medical College, Vellore, Tamil Nadu, India

Corresponding Author: Kiranmayi Govula, Department of Conservative Dentistry and Endodontics, Narayana Dental College and Hospital, Dr NTR University of Health Sciences, Nellore, Andhra Pradesh, India, Phone: +91 9440932608, e-mail: kiranmayigovula81@ gmail.com

How to cite this article: Govula K, Anumula L, Swapna S, et al. Interleukin-6: A Potential Salivary Biomarker for Dental Caries Progression-A Cross-sectional Study. Int J Experiment Dent Sci $2021 ; 10(1): 8-13$.

Source of support: Nil

Conflict of interest: None

predominant cell types within periapical lesions are neutrophils, macrophages, T \& B lymphocytes, mast cells, osteoclasts, osteoblasts, and fibroblasts. These cells express many pro-inflammatory cytokines, including IL-6, IL-4, IL-8, and TNF- $a$. These cytokines, when released into the systemic circulation, get accumulates within the periapical lesions. Among these, IL- 6 has been implicated in the pathology of various diseases because of its pleiotrophy. It plays a vital role in immunosenescence and dental caries. ${ }^{4}$ 
Immune processes affect the dental biofilm, which impacts caries susceptibility, and studies have evaluated components of innate and adaptive immune responses that affect caries risk. To date, several studies have explored the relationship between IL- 6 and systemic conditions such as lichen planus, aphthous stomatitis, periodontal problems. ${ }^{5}$ Though there is enough evidence of raised levels of IL- 6 in the saliva of patients with dental caries, there is no much research work regarding this correlation. This study has been attempted to explore a strong correlation between the levels of IL- 6 and dental caries.

Therefore, the individuals with dental caries may also be at high risk of having higher levels of IL-6. Thus by removing the cause for the inflammation that is dental caries, there can be a probability of reducing the IL- 6 levels. The present study aimed to observe whether there is any relation between dental caries and IL- 6 levels. The study aimed to test the hypothesis that there will be no change in IL- 6 levels in saliva after removing the carious lesions.

\section{Materials and Methods}

\section{Study Participants}

Twenty patients diagnosed with dental caries as the clinical finding was included in this study based on inclusion criteria,

- 18-39 years of age.

- ICDAS scores 1-5.

- Oral Hygiene Index-Debris Index scores are 0 and 1, and calculus index scores are 0 and 1.

And the Exclusion criteria were

- Patients with ICDAS score 5, oral hygiene index-debris index score is 2 and 3 , and calculus index score is 2 and 3.

- Medically compromised patients, patients with autoimmune disorders, pregnant patients, chronic smokers.

- Grossly decayed teeth that have to be extracted and presence of an abscess, sinus.

Twenty adults aged between 18 years and 40 years were diagnosed with dental caries with ICDAS 4 and 5 scores, and with 0 and 1 oral hygiene index scores were included in the study. Exclusion criteria included the patients with oral hygiene index 2 and 3 scores, autoimmune disorders, medically compromised, pregnant women, teeth with the presence of sinus tract, abscess, and teeth that had to be extracted.

\section{Experimental Design}

The study was conducted from July 2019 to September 2019 in the Department of Conservative Dentistry and Endodontics after getting approval from the institutional ethical committee [IEC $\mathrm{NDCH} / 2019 / \mathrm{P}-06]$. Each included patient was explained about the procedure, and informed consent was obtained before the study's commencement. Before treatment, a detailed case history followed by a thorough clinical examination and oral hygiene status was recorded for all the included patients. After collecting the first sample of saliva, treatment procedures like restorations oral hygiene procedures were performed. After a maintenance phase of 60 days, a second saliva sample was collected, and both the samples were subjected to an ELISA test for IL-6 level evaluation.

\section{Saliva Sample Collection}

The first saliva samples were collected by asking the patients to refrain from eating for 60 minutes. The individuals were asked to rinse their mouths 30 minutes before the collection. The saliva was then collected and transferred into Eppendorf tubes and was centrifuged at 4,500 rpm for 15 minutes to remove the mucin and debris that could interfere with the antigen-antibody reaction. After centrifugation, the supernatant saliva was separated into another Eppendorf tube and stored at the ultra-cool storage unit to prevent microbial growth and avoid cytokines' degeneration.

Based on the clinical examination and radiological investigations, we formulated the treatment plan, and treatment was performed as a chairside procedure. Treatments such as oral prophylaxis and restorative procedures were carried out. Every patient was recalled for a follow-up appointment to reinforce oral hygiene and the reassessment of restorations. After 60 days of the follow-up period, the second saliva sample was collected, centrifuged, and stored similarly to that of the first saliva sample. Both the samples were subjected to the ELISA test to determine the IL- 6 levels.

\section{ELISA Procedure and Analysis}

ELISA is a sandwich immunoassay with a precoated micro ELISA plate (antibody specific to human IL-6). First, the standards and the samples were added to the ELISA microplate wells. Biotinylated detection antibody, Avidin horseradish peroxidase conjugate (HRP), was added and incubated at $30^{\circ}$ for 30 minutes (Fig. 1). The wells that contained human IL- 6 biotinylated antibodies, the HRP, appeared in blue. The enzyme-substrate reaction was ended by adding a stop solution that turns into a yellow color (Fig. 2). The samples' intensity was directly proportional to the amount of cytokine bound to the captured antibody. Finally, the optical density (OD) was measured spectrophotometrically at $450 \pm 2 \mathrm{~nm}$ (Fig. 3). The OD values of the ELISA test were directly proportional to the IL- 6 concentration. The OD of the reaction was compared with the standard samples, and the ELISA reader program automatically excluded samples outside the reliable area of the standard curve.

\section{Statistical Evaluation}

The mean values of IL- 6 pre- and posttreatment were statistically analyzed using the Wilcoxon signed-rank test. Spearman's rankorder correlation test evaluated the correlation between the number of carious teeth and IL-6 levels.

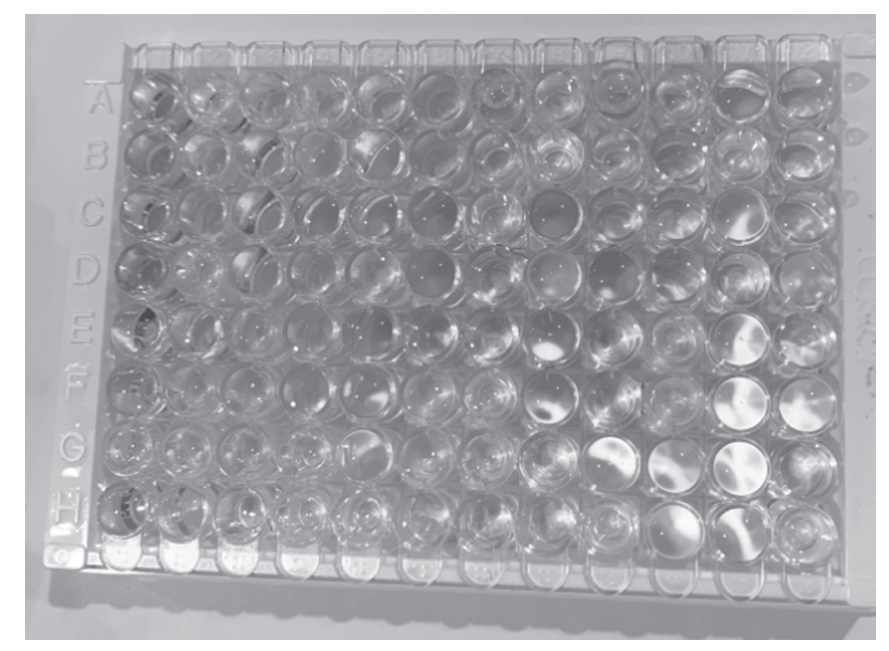

Fig. 1: After addition of HRP and biotinylated antibody 


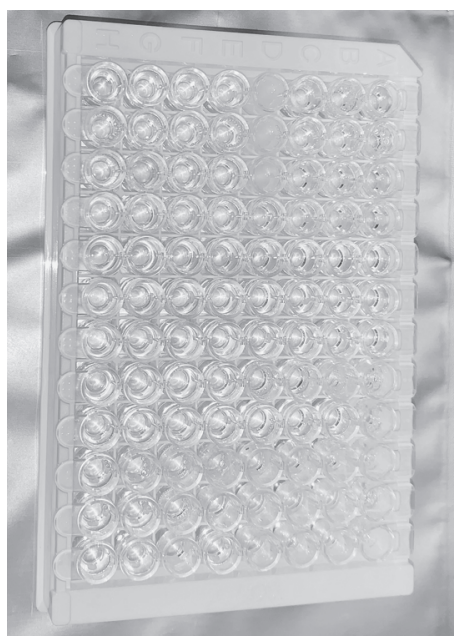

Fig. 2: Enzyme-substrate reaction was ended by adding a stop solution that turns into a yellow color

Table 1: Demographic characteristics

\begin{tabular}{|c|c|c|}
\hline & Frequency $(n)$ & Percentage \\
\hline \multicolumn{3}{|l|}{ Gender } \\
\hline Male & 10 & 50 \\
\hline Female & 10 & 50 \\
\hline \multicolumn{3}{|l|}{ Age (in years) } \\
\hline $18-29$ & 11 & 55 \\
\hline $30-40$ & 09 & 45 \\
\hline \multicolumn{3}{|l|}{ Location } \\
\hline Urban & 08 & 40 \\
\hline Rural & 12 & 60 \\
\hline \multicolumn{3}{|l|}{ Occupation } \\
\hline Professional & 08 & 40 \\
\hline Student & 04 & 20 \\
\hline Housewife & 08 & 40 \\
\hline \multicolumn{3}{|l|}{ Diet } \\
\hline Vegetarian & 01 & 5 \\
\hline Mixed & 19 & 95 \\
\hline \multicolumn{3}{|l|}{ Type of brushing } \\
\hline Paste and brush & 20 & 100 \\
\hline Others & 0 & 0 \\
\hline \multicolumn{3}{|l|}{ Type of brush } \\
\hline Medium & 15 & 75 \\
\hline Soft & 05 & 25 \\
\hline \multicolumn{3}{|l|}{ Brushing method } \\
\hline Horizontal & 19 & 94 \\
\hline Others & 01 & 6 \\
\hline \multicolumn{3}{|c|}{ Frequency of brushing } \\
\hline Once & 16 & 80 \\
\hline Twice & 04 & 20 \\
\hline
\end{tabular}

\section{Results}

The study included 20 patients (10 males and 10 females) who reported in the outpatient department seeking dental treatment in the OPD of the Department of Conservative Dentistry and Endodontics at Narayana Dental College and

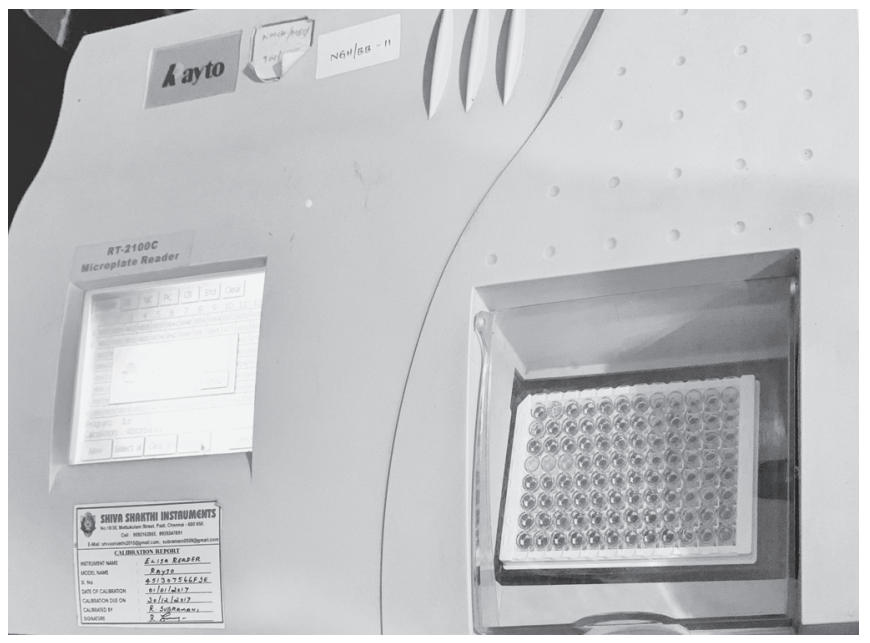

Fig. 3: Optical density is read on a standard plate reader at $450 \mathrm{~nm}$

Table 2: Distribution of sample based on gender, age and location

\begin{tabular}{|c|c|c|c|c|}
\hline $\begin{array}{l}\text { Clinical pa- } \\
\text { rameters }\end{array}$ & Mean $\pm S D$ & $\begin{array}{l}\text { Mean } \\
\text { difference }\end{array}$ & tvalue & pvalue \\
\hline \multicolumn{5}{|c|}{ Gender } \\
\hline \multicolumn{5}{|l|}{ Pretreatment } \\
\hline Males & $16.66 \pm 20.49$ & 2.672 & 0.332 & $0.743^{* *}$ \\
\hline Females & $13.98 \pm 15.03$ & & & \\
\hline \multicolumn{5}{|l|}{ Posttreatment } \\
\hline Males & $4.29 \pm 1.50$ & 0.209 & 0.314 & $0.757^{* *}$ \\
\hline Females & $4.08 \pm 1.47$ & & & \\
\hline \multicolumn{5}{|c|}{ Age } \\
\hline \multicolumn{5}{|l|}{ Pretreatment } \\
\hline 18-29 Years & $17.30 \pm 19.07$ & 4.394 & 0.547 & $0.591^{* *}$ \\
\hline 30-40 Years & $12.90 \pm 16.25$ & & & \\
\hline \multicolumn{5}{|l|}{ Posttreatment } \\
\hline 18-29 Years & $4.16 \pm 1.37$ & -0.068 & -0.102 & $0.920^{* *}$ \\
\hline 30-40 Years & $4.23 \pm 1.61$ & & & \\
\hline \multicolumn{5}{|c|}{ Location } \\
\hline \multicolumn{5}{|l|}{ Pretreatment } \\
\hline Urban & $19.59 \pm 22.10$ & 7.118 & 0.884 & $0.388^{* *}$ \\
\hline Rural & $12.47 \pm 14.09$ & & & \\
\hline \multicolumn{5}{|l|}{ Posttreatment } \\
\hline Urban & $3.09 \pm 1.10$ & 0.868 & 1.339 & $0.197^{* *}$ \\
\hline Rural & $3.93 \pm 1.28$ & & & \\
\hline
\end{tabular}

Hospital. Recruitment was based on the clinical examination and radiographical interpretation (inclusion criteria). The study characteristics, along with the clinical variables, are given in detail in Table 1.

Table 2 shows the distribution of the sample based on the age group and location. The included population ranges from 18 to 40 years (11 belonged to 18-29 years, and 9 belonged to 30-40 years). Based on the location, 8 were from urban areas, and 12 were from rural areas. The independent sample $T$-test showed no statistical significance $(p<0.05)$ difference between variables like gender, age, location, and pre- and posttreatment IL- 6 levels. 
IL-6 Levels before and after Treatment

Table 3: Distribution of sample based on occupation

\begin{tabular}{|c|c|c|c|c|}
\hline $\begin{array}{l}\text { Clinical } \\
\text { parameters }\end{array}$ & Mean $\pm S D$ & Mean ranks & $\begin{array}{l}\text { Mann- } \\
\text { Whitney value }\end{array}$ & $p$ value \\
\hline \multicolumn{5}{|c|}{ Type of brush } \\
\hline \multicolumn{5}{|c|}{ Pretreatment } \\
\hline Medium & $14.50 \pm 16.88$ & 10.20 & 33.000 & $0.694^{* *}$ \\
\hline Soft & $17.79 \pm 21.29$ & 11.40 & & \\
\hline \multicolumn{5}{|c|}{ Posttreatment } \\
\hline Medium & $3.94 \pm 1.40$ & 9.60 & 24.000 & $0.238^{* *}$ \\
\hline Soft & $4.92 \pm 1.49$ & 13.20 & & \\
\hline \multicolumn{5}{|c|}{ Frequency of brushing } \\
\hline \multicolumn{5}{|c|}{ Pretreatment } \\
\hline Once & $14.64 \pm 16.24$ & 10.72 & 28.500 & $0.741^{* * *}$ \\
\hline Twice & $18.04 \pm 24.84$ & 9.63 & & \\
\hline \multicolumn{5}{|c|}{ Posttreatment } \\
\hline Once & $4.43 \pm 1.31$ & 11.47 & 16.500 & $0.143^{* *}$ \\
\hline Twice & $3.24 \pm 1.78$ & 6.63 & & \\
\hline
\end{tabular}

Mann-Whitney UTest: ${ }^{*} p<0.05$ (significant), ${ }^{* *} p>0.05$ (not significant)

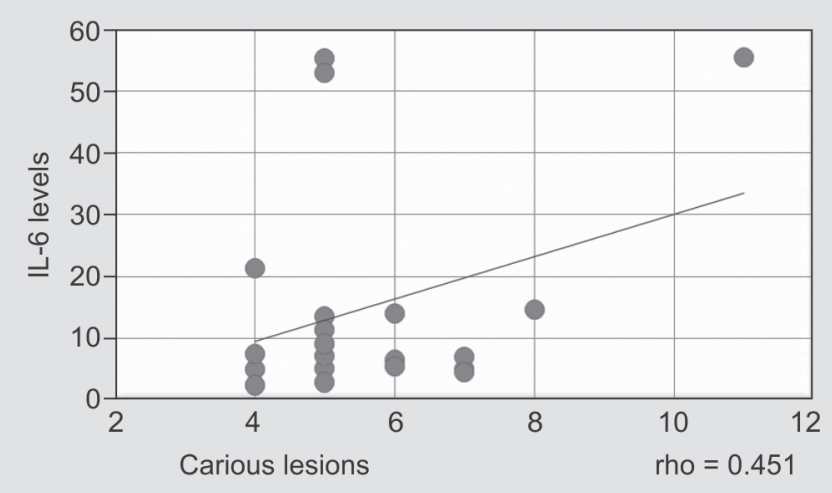

Fig. 4: Pretreatment-Spearman's rank-order correlation

Table 3 shows the mean ranks according to the Mann-Whitney test for pre-and posttreatment levels of IL 6 according to the type of brush used, frequency of brushing. There exists a slight dissimilarity that is not statistically significant.

The Kruskal-Wallis test showed no statistically significant $(p \leq$ 0.001 ) difference for the category of the type of brush used and the frequency of brushing regarding pre- and posttreatment levels of IL-6 shown in Table 4.

Table 5 shows the pre- and posttreatment; IL- 6 levels were statistically analyzed using the Wilcoxon signed-rank test. The pretreatment group's mean and SD values were $15.32 \pm 17.54$, and for the posttreatment group, it was $4.19 \pm 1.45$. Furthermore, the $p$ value was $<0.001$, which infers a statistically significant difference between pre- and posttreatment IL- 6 levels. The correlation between the number of carious teeth and IL-6 levels was evaluated using Spearman's rank-order correlation test mentioned in Figures 4 and 5. Figures 4 and 5 show Spearman's rank-order correlation between carious lesions and IL-6 levels for both pre- and posttreatment. Spearman's correlation will determine the strength and direction of the monotonic relationship between two variables.

Figure 4 shows a positive correlation between the carious lesion and IL- 6 levels, confirming a direct relationship between and two. In this figure, there is a general upward trend, which implies a positive correlation. If we plug the data, which implies a positive
Table 4: Distribution of sample based on oral hygiene habits

\begin{tabular}{|c|c|c|c|c|}
\hline $\begin{array}{l}\text { Clinical } \\
\text { parameters }\end{array}$ & Mean $\pm S D$ & Mean ranks & $\begin{array}{l}\text { Chi-square } \\
\text { value }\end{array}$ & $p$ value \\
\hline \multicolumn{5}{|c|}{ Occupation } \\
\hline \multicolumn{5}{|l|}{ Pretreatment } \\
\hline Professional & $18.35 \pm 22.42$ & 10.00 & 0.840 & $0.657^{* * *}$ \\
\hline Student & $7.98 \pm 4.58$ & 8.75 & & \\
\hline Housewife & $15.96 \pm 16.79$ & 11.88 & & \\
\hline \multicolumn{5}{|l|}{ Posttreatment } \\
\hline Professional & $4.10 \pm 1.48$ & 9.94 & 0.445 & $0.801^{* *}$ \\
\hline Student & $3.78 \pm 1.23$ & 9.50 & & \\
\hline Housewife & $4.48 \pm 1.62$ & 11.56 & & \\
\hline
\end{tabular}

Table 5: Pre- and post-score measurement of optical density of IL-6

\begin{tabular}{lcll}
\hline Time interval & Mean $\pm S D$ & Mean difference & $p$ value \\
\hline Pre & $15.32 \pm 17.54$ & 11.13 & $0.01^{*}$ \\
Post & $4.19 \pm 1.45$ & & \\
\hline
\end{tabular}

${ }^{*} p$ value was computed using Wilcoxon signed-rank test

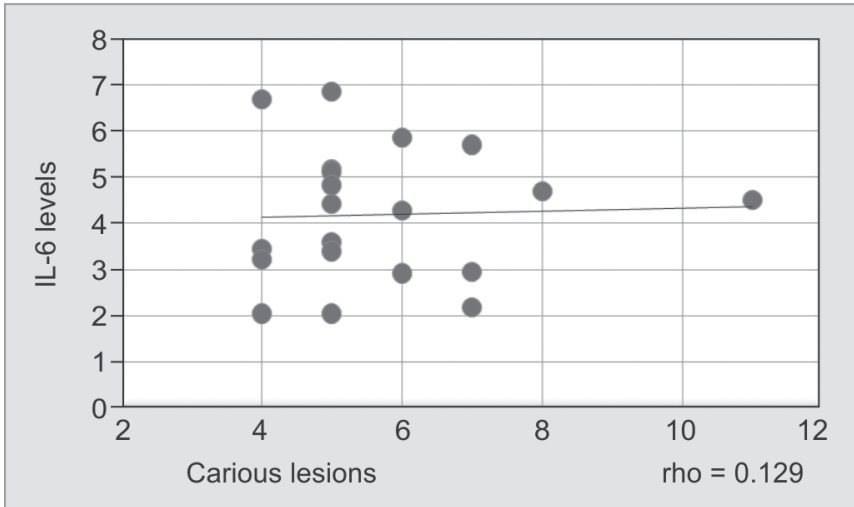

Fig. 5: Posttreatment-Spearman's rank-order correlation

correlation of determination into the coefficient of determination $r=0.452$, there is a moderate correlation between the variables.

Figure 5 represents Spearman's correlation in the posttreatment group, and IL- 6 represents a less correlation. The scatter plot's best fit for this data has a slope showing that correlation becomes weaker and moving close to zero.

Figure 6 represents the mean values of IL- 6 in pretreatment and posttreatment groups.

\section{Discussion}

The destruction of the dental tissues by microorganisms favors the development of dental caries, which further becomes the leading cause of pulpal inflammation. The constant increase in sugar concentration in food leads to the bacteria's adaptive changes in dental plaque, making the bacteria acid-resistant and acidforming and contributing to high caries production. ${ }^{6}$ Along with the microorganisms, the host immune response also plays a pivotal role in tissue destruction. The action of IL- 6 in pulpal inflammation was correlated in a study by Samad et al., 1994, which demonstrated the relationship between tissue destruction and IL-6. The reason may be that IL- 6 produced by dental pulp cells is involved in the 


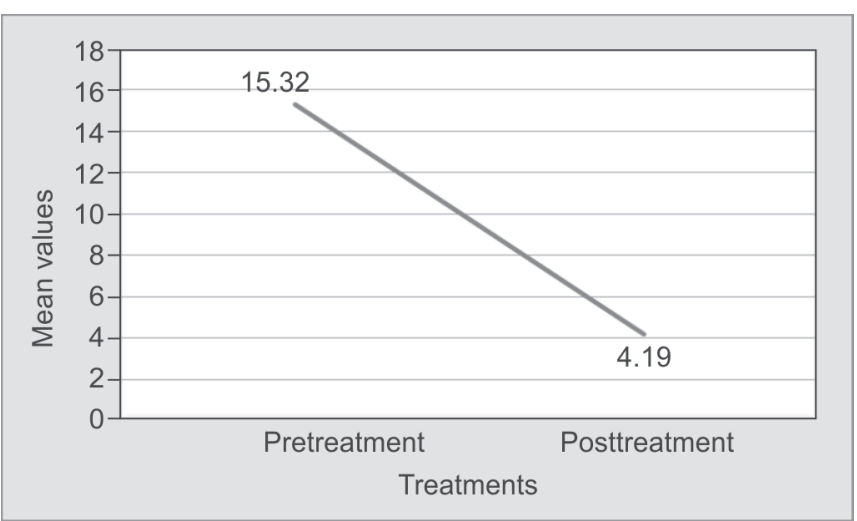

Fig. 6: Monotonic relationship between IL-6 and before and after caries removal

metabolism of the extracellular matrix and the destruction of the dental pulp tissue. ${ }^{7}$

Kurtis and Tuter in 2005 proved that higher levels of IL- 6 and TNF- $\alpha$ in patient's saliva with dental caries lead to a decrease in the number of osteoblasts and fibroblasts. ${ }^{8}$ Moreover, it also supports the demineralization of teeth, further leading to the development of dental caries. ${ }^{9}$

Further immunohistological observational studies are needed to evaluate the complete and detailed action of IL-6. The purpose of therapy or treatment is to restore the dynamic balance of proinflammatory or destructive mediators and anti-inflammatory or protective mediators similar to that of healthy individuals. ${ }^{10}$

Still, the molecular mechanisms of dental caries are unknown. The pro-inflammatory cytokines are said to play a vital role in such molecular mechanisms. However, research studies are to be done to explain in detail their mode of action. Inflammatory cytokines mediate or enhance inflammation and act as guiding factors for inflammation. They are potential targets for new therapeutic strategies in the treatment of inflammation or allergic diseases. Also, their levels can be correlated with the progression of bacterial infection. Hence, IL- 6 secretion in saliva will be a useful tool for diagnosing and monitoring dental caries disease. ${ }^{11}$

Salivary diagnostics is dynamic and emerging, utilizing nanotechnology and molecular diagnostic methods to diagnose oral and systemic diseases to measure biomarkers' release during disease initiation and progression. ${ }^{12}$

In this study, saliva was selected for assessing the levels of IL-6 as the collection procedure is non-invasive, and sample procurement is painless, reducing the patient discomfort and safer handling of the samples. Today, there are many methods available for quantifying the levels of IL- 6 in saliva. ELISA sandwich immunoassay was chosen as it is a simplified assay with increased sensitivity and specificity. It is a good option for a limited sample size and is best suited to quantify cytokine levels in saliva samples.

Nowadays, science strives to identify and monitor biomarkers to capture the various and complex processes. A biomarker is a traceable substance, indicating a change in the protein expression that correlates with the risk or disease progression or a condition's susceptibility to a given treatment. ${ }^{13}$ Such excellent biomarkers in saliva are inflammatory cytokines, among which IL- 6 has shown a strong correlation with pulpal inflammation and immunosenescence with dental caries. Inflammation and immune induction can be viewed as intimately linked events like the successful resolution of the inflammatory process leading to the transition from innate to adaptive immunity. An essential feature of IL- 6 is that it is implicated in regulating lymphocyte trafficking through lymph nodes following an inflammatory stimulus. ${ }^{14}$

ICDAS criteria were considered in this study for including adult patients with dental caries. It helps in understanding the process of initiation and progression of dental caries in epidemiological and clinical research. This system indeed allows us to record the severity and incidence of caries in its continuum.

A baseline study conducted by Gonowiez et al. compared the levels of various cytokines in children and adult patients with dental caries with that of healthy individuals and found that among all the cytokines, salivary IL-6 levels were significantly higher in both the groups with dental caries. After observing the results of various studies, IL-6 strongly correlates with the severity and extent of caries in the oral cavity. So minimizing the inflammation will lower or reduce the levels of IL-6 also. ${ }^{10,15}$

This study is a pioneering approach to assess the levels of salivary IL- 6 in adult patients with dental caries before and after caries removal or caries treatment. In this study, the prevalence of dental caries is higher in the younger age group of 18 to 29 years than in the other group. Hence, it is essential to develop awareness about caries in young patients, educate them about the dietary habits, brushing method, and duration and frequency of brushing. Participants from rural areas showed a higher prevalence of dental caries (60\%) than participants from urban areas (40\%). The number of decayed teeth was slightly higher in females than males, and the number of decayed mandibular molars was higher than maxillary molars. This finding may be because the mandibular first molar eruption precedes the eruption of the maxillary molar.

Figures 4 and 5 show Spearman's rank-order correlation between carious lesions and IL- 6 levels for both pre- and posttreatment. Spearman's correlation determines the strength and direction of the monotonic relationship between two variables. Figure 4 shows a general upward trend, which implies a positive correlation means the Scatter plot proves that the value of one variable increases values of another variable also increases.

Figure 5 represents Spearman's correlation in the posttreatment group, and IL- 6 represents a weaker correlation. The line of best fit in the scatter plot for this data has a slope showing that correlation becomes weak due to the irritant caries removal. After removing the carious lesions and restoring the teeth with permanent restorations and oral hygiene maintenance, it gradually made the correlation weaker. The correlation is confirming once again that there is a direct relationship that exists between caries and IL- 6 levels.

Figure 6 represents the mean values of IL- 6 in pretreatment and posttreatment groups. The figure shows a significant reduction in the mean value from the pretreatment group to the posttreatment group. The clinical variables such as gender, age factor, location, occupation, and brushing habits have not affected IL- 6 before and after caries removal. Only complete removal of caries has shown a reduction in the IL- 6 values.

Inflammatory cytokines are the potential targets for new therapeutic strategies in treating inflammatory diseases. ${ }^{16}$ The pre- and posttreatment interleukin levels of optical densities were analyzed by Wilcoxon signed-rank test. The mean salivary IL-6, as estimated in this study, was $15.32 \pm 17.54$, which significantly reduced to $4.19 \pm 1.45$ after posttreatment. This notable reduction of IL- 6 values can be attributed to the complete removal of dental caries, resulting in restorative therapies. Further studies with a larger sample size at a longer follow-up period may help to draw new 
conclusions and in establishing the efficacy of dental treatments in minimizing the levels of these inflammatory markers.

\section{Conclusion}

There is a strong correlation between IL- 6 levels and the extent and severity of carious lesions. The study was an initial effort to evaluate the relationship between salivary IL- 6 and dental caries and study the effect of dental treatments on reducing it in the oral cavity. The levels of IL-6 reduced significantly after the complete removal of caries and restoring those teeth with restorative materials.

\section{Statement of Ethics}

The study got approval from the institutional ethical committee [IEC NDCH/2019/P-06].

\section{Authors Contribution}

Dr Kiranmayi principle investigator-Study design, methodology, manuscript writing

Dr Lavanya co-investigator-Manuscript editing

Dr Swapna-Manuscript writing and editing

Mr. Richard Kirubakaran-Statistical analysis.

\section{ACKnowledgments}

We want to thank the contribution of biochemists, lab technicians, senior staff members, and colleagues.

\section{References}

1. Aziz AR, Mohammed AT. The salivary inflammatory biomarkers (Interleukin-6, C-reactive protein) in relation with caries-experience among a group of 12 year old obese boys. J Baghdad Coll Dentis 2016;28(1):138-142. DOI: 10.12816/0024723.

2. Sruthi KS, Yashoda R, Manjunath PP. Diagnostic potential of saliva as a biomarker in early childhood caries: a review. Children 2019; 11(12):14.

3. Nakahara $\mathrm{H}$, Song J, Sugimoto $M$, et al. Anti-interleukin- 6 receptor antibody therapy reduces vascular endothelial growth factor production in rheumatoid arthritis. Arthritis Rheum 2003;48(6):15211529. DOI: 10.1002 /art.11143.
4. Guo L, Shi W. Salivary biomarkers for caries risk assessment. California Dent Associat 2013;41(2):107.

5. Hemadi AS, Huang R, Zhou Y, et al. Salivary proteins and microbiota as biomarkers for early childhood caries risk assessment. Int J Oral Science 2017;9(11):e1. DOI: 10.1038/ijos.2017.35.

6. Roi A, Rusu LC, Roi Cl, et al. A new approach for the diagnosis of systemic and oral diseases based on salivary biomolecules. Dis Mark 2019;2019:8761860. DOI: 10.1155/2019/8761860.

7. Simpson RJ, Hammacher A, Smith DK, et al. Interleukin-6: Structurefunction relationships. Prot Sci 1997;6(5):929-955. DOI: 10.1002/ pro. 5560060501 .

8. Kurtiş B, Tüter G, Serdar M, et al. Gingival crevicular fluid levels of monocyte chemoattractant protein-1 and tumor necrosis factor-alpha in patients with chronic and aggressive periodontitis. J Periodontol 2005;76(11):1849-1855. DOI: 10.1902/jop.2005.76. 11.1849.

9. Elsalhy M, Azizieh F, Raghupathy R. Cytokines as diagnostic markers of pulpal inflammation. Int Endodon J 2013;46(6):573-580. DOI: 10.1111/ iej.12030.

10. Gornowicz A, Bielawska A, Bielawski K, et al. Pro-inflammatory cytokines in saliva of adolescents with dental caries disease. Ann Agricult Environ Med 2012;19(4):711-716

11. Hosoya S, Matsushima K, Ohbayashi E, et al. Stimulation of interleukin$1 \beta$-independent interleukin- 6 production in human dental pulp cells by lipopolysaccharide. Biochem Mole Med 1996;59(2):138-143. DOI: 10.1006/bmme.1996.0079.

12. Menon MM, Balagopal RV, Sajitha K, et al. Evaluation of salivary interleukin- 6 in children with early childhood caries after treatment. Contemp Clin Dentis 2016;7(2):198. DOI: 10.4103/0976-237X. 183059.

13. Lalloo R, Tadakamadla SK, Kroon J, et al. Carious lesions in permanent dentitions are reduced in remote indigenous Australian children taking part in a non-randomised preventive trial. PLOS ONE 2021;16(1):e0244927. DOI: 10.1371/journal.pone.0244927.

14. Devaraj SD. Salivary biomarkers-a review. J Pharmaceut Sci Res 2013;5(10):210.

15. Biomarker (medicine) - Wikipedia. https://en.wikipedia.org/wiki/ Human_diseases_marker.

16. Kopf M, Bachmann MF, Marsland BJ. Averting inflammation by targeting the cytokine environment. Nat Rev Drug Discov 2010;9(9):703-718. DOI: 10.1038/nrd2805. 